\title{
The insulin resistance syndrome: implications for thrombosis and cardio- vascular disease
}

\author{
Irène Juhan-Vague, Pierre E. Morange, Marie-Christine Alessi \\ Lab. Haematology. CHU Timone. Inserm EPI 9936 . Marseille . France
}

\section{Key Words}

Insulin resistance - Obesity - Inflammation Atherothrombosis - Endothelial cells - Platelets Clotting factors - PAI-1

\begin{abstract}
The insulin resistance syndrome ( IRS) with obesity is large-word wide-spread and represents a strong risk factor for vascular disease. Atherothrombotic complications in IRS are partly attributed to a dysregulation of hemostasis inducing a prothrombotic state which includes endothelial activation, hyperactivity of platelets, hypercoagulability and hypofibrinolysis. This latter, due to elevated PAI-1 levels, is a core feature of the IRS. Most of the prothrombotic modifications can be reversed by loosing weight. Low grade inflammation with prolonged cytokines mediated acute phase reaction is actually considered as strongly related to the IRS and is involved in the dysregulation of hemostasis. TNF pathway and TGFb play an important role in the regulation of PAI-1 synthesis in the adipose tissue and the liver with steatosis. Interestingly, modulation of PAl-1 expression in adipose tissue influences adipose tissue growth, increasing once more the spectrum of the non hemostatic functions of coagulation/fibrinolysis parameters.
\end{abstract}

Copyright (C) 2002 S. Karger AG. Basel

\section{The insulin resistance syndrome}

The insulin resistance syndrome, also called metabolic syndrome or syndrome $\mathrm{X}$, is defined by a cluster of abnormalities that have been implicated in acceleration of atherothrombosis. Components of the metabolic syndrome include obesity with a repartition of the fat in the central part of the body (visceral or android obesity), glucose intolerance, hypertension, hyperinsulinemia, dyslipidemia with elevated triglyceride and lowered HDL cholesterol concentration, and increased proportion of small dense lipoparticules. Very interestingly, a prothrombotic state has also recently been described.

Low insulin sensitivity may have multiple origins, among them genetic molecular defects in pathways of cellular insulin effects but it is mainly triggered by energetic imbalance due to increased energy-dense food consumption and reduced daily physical expenditure. The portal/visceral hypothesis was proposed in which increased adiposity, particularly in the visceral depots, leeds to increased free fatty acid flux and inhibition of insulin action in insulin sensitive tissues. The insulin resistance syndrome could represent the failure of the adipose tissue mass to expand and thus to accommodate an increased energy influx. Increased adipocyte size is prevented by insulin resistance and this contributes to deviate lipid through other organs such as liver, muscle, pancreas and possibly vascular wall (1).

Insulin resistance is large world wide-spread. Its prevalence in USA is around $23 \%$ and increases from $7 \%$ for participants aged 20 through 29 years to $43 \%$ for participants aged 60 through 69 years (2). Moreover, the prevalence of obe-

Irène Juhan-Vague

Laboratory of Haematology, CHU Timone, 13385 Marseille Cedex 5, France tel: 33491492449

fax: 33491942332

E-Mail: ijuhan@ap-hm.fr 
sity has increased for $20 \%$ in the last 10 years in the majority of the western countries. People with the metabolic syndrome are at increased risk for developing diabetes mellitus and cardiovascular disease. The presence of this syndrome increases by a factor 6 the cardiovascular mortality in a follow up of 6.9 years (3). Fasting insulin levels reasonably estimate insulin resistance measured by direct assessment, specially among subjects with normal glucose tolerance, and fasting insulin has been reported to be an independant risk factor for coronary heart disease (4).

Much effort is made on revealing the mechanisms behind the increased risk of cardiovascular disease observed in the insulin resistance syndrome. The particular dyslipidemia along with hyperinsulinemia, and hypertension are implicated. More and more interest is actually focused on the description of a prothrombotic state, as there is an accumulating body of evidence that the hemostatic system is involved in the cardiovascular disease process.

\section{Dysregulation of hemostasis in insulin resistance}

An abundant litterature has now established that insulin resistant patients present a prothrombotic state.

Disturbances of the hemostatic system may favor the development of vascular damage, by playing a role in the vessel wall remodelling and the final occlusion event, by promoting thrombosis at the site of a suddendly ruptured atherosclerotic plaque. Changes in the hemostatic system may have several aspects which are often encountered simultaneously : - activation of endothelial cells and leukocytes, - hyperactivity of platelets, - hypercoagulability with increased formation of the multipotent thrombin, - high levels of fibrinogen, which promotes platelet aggregation and induces rheological changes and the formation of a rich fibrin clot, - decreased fibrinolytic activity due to an increased plasma level of the PAI-1, which leads to decreased removal of fibrin deposits and matrix accumulation in the vessel wall. All of these changes can be observed in obese insulin resistant subjects. However increased PAI-1 expression with the resultant hypofibrinolysis is the main hemostasis disorder linked to insulin resistance and is now considered as part of the cluster of abnormalities of the metabolic syndrome (5).

The mechanisms that induce a prothrombotic state in insulin resistant obese patients are multifactorial. In vitro studies have demonstrated that insulin and free fatty acid, which plasma concentration increase during insulin resistance, can directly affect platelet functions or endothelial cells or the hepatocytes synthesis of hemostatic factors.

Low grade inflammation with prolonged cytokines mediated acute phase reaction is actually considered as strongly relat- ed to the insulin resistant syndrome state. Adipose tissue is an important source of IL6 and TNF has been shown to be overexpressed in the adipose tissue of obese insulin resistant patients. In clinical studies both obesity, atherosclerosis and diabetes are associated with elevated plasma levels of IL6 and CRP. Thus a prolonged cytokines- mediated acute phase reaction may be part of a wide-ranging innate immune response which through the action of cytokines on the hepatocytes, monocytes-macrophages, endothelial cells, adipose tissue, contributes to the biochemical and clinical features of the metabolic syndrome (6) and to the development of atherothrombotic lesions. Furthermore inflammation may trigger insulin resistance (7) and this may be a common precursor of both atherosclerosis, cardiovascular disease and diabetes. Nevertheless the development of insulin resistance is subjected to large interindividual variability suggesting the presence of a complex interplay between genetic susceptibility and environment.

We are going to review the changes of the hemostatic system in insulin resistant obese patients. Interestingly it appears clearly that some changes are well related to obesity per se whereas other changes are more strickly associated with the features of the metabolic syndrome.

\section{1 - Endothelial hemostatic dysfunction}

An endothelial activation has been described in obese patients, with an increase in circulating intercellular adhesion molecule 1 (ICAM1) and vascular adhesion molecule 1 (VCAM1). Vascular responses to L Arginine, the natural precursor of nitric oxide (NO), was also impaired in obese compared to normal weight women. These changes were shown to be correlated with visceral body fat and circulating cytokines such as TNF and IL6 and were ameliorated by weight loss (8).

The plasma concentration of von Willebrand factor (vWF) has been studied by several groups. Among men and women in the Framingham offspring study, vWF was positivelly associated with insulin levels among subjects with normal glucose tolerance (9). VWF is, therefore, weakly related to the metabolic factors involved in the insulin resistance syndrome, but rather, as fibrinogen, to the inflammatory process which accompanies obesity per se. Indeed, vWF is a marker of the acute phase reaction and is positively correlated with fibrinogen or CRP levels (10).

\section{2 - Hyperactivity of platelets}

Insulinresistance could be directly implicated in hyperactivity of platelets. Insulin administered in vivo inhibits the platelet interaction with collagen under conditions mimicking thrombus formation and reduces aggregation to several agonists of platelets from normal subjects but not from obese insulin resistant patients (11). Interestingly, it was recently demonstrated that leptin, an adipose tissue synthesized protein, which is increased in the plasma of the obese subjects, induces 
platelet aggregation via leptin receptors on platelet membrane (12).

\section{3 - Hypercoagulability}

The increased concentration of fibrinogen is a consistently described plasma abnormality in obesity, but is inconsistently associated with glucose intolerance or with hyperinsulinemia in non diabetic subjects. It is now considered that the fibrinogen level is determined by overall adiposity rather than insulin resistance (13). It is related to glycemic control and to obesity per se and weakly related to abdominal obesity and insulin levels. IL6 is thought to be at the basis of this abnormality. IL6 is produced by adipose tissue (14) and will directly stimulate the hepatic synthesis of fibrinogen. IL6 could therefore represents a link between obesity and fibrinogen level in plasma.

The plasma levels of other clotting factors are elevated in obesity such as Factor VIII or Factor VII. Several studies have documented reversal of numerous disturbances in the plasma coagulation cascade induced by loosing weight (15).

\section{4 - Hypofibrinolysis}

It is now well established that hypofibrinolysis due to elevated plasma PAI-1 levels is a core feature of the insulin resistance syndrome.The mechanisms of overexpression of PAI-1 should be distinguished from that of fibrinogen or vWF. PAI-1 levels are elevated in obese insulinresistant patients as well as in non-insulin dependent diabetic patients and are in the normal range in type 1 diabetic patients. Plasma PAI- 1 levels have been shown for a long time to be associated with the parameters of the insulin resistance syndrome (5). They correlate with BMI, visceral fat ,which characterizes android obesity, blood pressure, plasma levels of insulin or proinsulin, triglycerides, small dense LDL particules, free fatty acids, and are inversely related with HDL cholesterol, whereas no correlation is observed to measures of glycemic control (for review see 16).

The modulation of insulinresistance by hypocaloric diet with loosing weight, physical training, change in dietary composition, the use of oral antidiabetic drugs such as metformin, induce a decrease in PAI-1 levels which correlate with the decrease in weight and in plasma metabolic parameters (15, 16). It was shown recently that the use of thiazolidinediones such as troglitazone is associated with a decrease in plasma PAI-1 levels in persons with type 2 diabetes (17). Whereas insulin added in vitro to hepatocytes induces an increase PAI1 synthesis, and hyperinsulinemia is associated with elevated plasma PAI-1 levels , an acute administration of insulin does not modify plasma PAI-1 concentration (16).

One uncertainty about PAI-1 synthesis during insulin resistance is its cell origin. Unequivocally many cells/tissues could synthesize PAI-1. But since PAI-1 gene expression is inducible rather than constitutive, one of the foremost question is to recognize the inducer which is concerned in insulin resistance.
One approach was to propose that metabolic disturbances observed during this syndrome, excess of insulin, triglycerides, free fatty acid, angiotensin II, directly affect PAI-1 synthesis. Indeed, most cell culture experiments confirm these hypothesis. And it has been shown that insulin, glucocorticoids, VLDL, free fatty acid, glucose, angiotensin II increase the PAI-1 production by cells in culture (16). And despite the fact that clinical observations do not always support such a direct effect, interesting concepts have emerged.

Among the other potential inducers of PAI-1 synthesis, the pro-inflammatory cytokines TNF appears of particular relevance in the context of insulin resistance. Indeed, it has deleterious effects on both glucose homeostasis and beta-cell function, and can disrupt insulin signalling pathways in both pancreatic beta cells and liver and adipose tissue (7). In mice the adipose tissue revealed to contain high amount of PAI-1 and the invalidation of both TNF receptors results in a significantly reduced adipose tissue PAI-1 expression and plasma PAI-1 levels (18).

We have described (19), as others, a PAI-1 synthesis by human adipose tissue. We have shown a decrease of PAI-1 expression in adipose tissue by weight loss and a variability of PAI-1 expression depending on fat territory. Visceral fat produces more PAI-1 than subcutaneous or femoral fat. The main PAI-1 producing cells were identified as stroma cells, some of them being of monocyte or smooth muscle cell origin. Thus, the adipose tissue could represent a PAI-1 reservoir. However these results do not exclude the contribution of other tissues. Indeed, the liver is also a good candidate since some groups have observed a strong relationship between plasma PAI-1 and liver steatosis levels. We found that the PAI-1 antigen content in human adipose tissue was highly correlated with those of TNF receptors and TGFb, but not to that of TNF or IL6 which were both strongly correlated to each other. These results from mice and human studies suggest that the TNF pathway could be a common link between elevated PAI-1 and insulin resistance. On another hand, the levels of TGFb, one of the strongest inducers of PAI-1 synthesis in vitro are also associated with those of PAI-1 within the human adipose tissue and could be involved in the regulation of PAI-1 during insulin resistance.

What are the consequences of PAI-1 overexpression in insulin resistance?

The high expression of PAI-1 during insulin resistance queries on the finality of this phenomenon. PAI- 1 is involved in tissue remodelling, as it interferes, via its binding to vitronectin, to the cellular adhesion, migration and proliferation processes. Therefore, it can be suggested that the elevated expression of PAI-1 observed in obesity is involved in adipose tissue development. The modulation of the PAI-1 gene in mice fed with high fat diet induces change in weight gain : PAI-1 KO mice gain weight faster (20) whereas PAI-1 transgenic 
mice with an overexpression of PAI-1 gain less weight than their respective wild couterparts (21). On the same line, it was recently shown that plasminogen $\mathrm{KO}$ mice fed high fat diet gained less weight. And in vitro experiments support the fact that the hypofibrinolysis induced by excess of PAI- 1 or the absence of plasminogen leads to a decrease of preadipocytes differenciation with consequences on the adipose tissue growth. The PAI-1 overexpression in insulin resistance could control the fat mass mainly by reducing adiposity. Involvment of PAI-1 to insulin resistance requers further investigations.

Despite this suspected protective effect on obesity, and perhaps deleterious effect on insulin resistance, an excess of PAI1 could increase the rate of atherosclerosis and diabetes. High plasma PAI-1 levels have been considered as a risk factor for CHD (22). PAI-1 has been shown to be predictive of events in univariate analysis. However the predictivity disappeared after adjustment for BMI, triglycerides, HDL cholesterol, markers of insulin resistance. Elevated levels of PAI-1 and insulin have been associated with increased artery intimal-medial thickness in non diabetic subjects. From these data it could be speculated that increased plasma PAI-1 levels are the witness of insulin resistance; however, some in vitro data argued for a specific role of PAI-1 in vascular lesion development. Results were mostly obtained in genetically modified animals. Sobel et al (23) have provided evidence that type II diabetes is associated with an increased PAI-1 expression in the arterial wall. This increased PAI-1 concentration in the vessel wall could participate, as well as the increased plasma PAI-1 level, in the accelerated vasculopathy of these patients.
Very interestingly, it was recently demonstrated in a large cohort of healthy non diabetic subjects, that patients who develop an incident diabetes within 5 years presented higher levels of fibrinogen, CRP and PAI-1 at baseline than non converters (24). PAI-1 predicted type 2 diabetes independently of insulin resistance and other known risk factors for diabetes.

In this line, PAI-1 could play a part in the development of cardiovascular diseases, and in type 2 diabetes. Both these properties indicate that PAI-1 represent an ideal target for therapeutic intervention that aims to decrease the risk of both cardiovascular disease and type 2 diabetes.

\section{Conclusion}

A procoagulant state has been described in obese insulin resistant patients and type 2 diabetic patients. Obesity per se and insulin resistance both contribute to this deleterious effect. Interestingly PAI-1 could be involved in the genesis of this metabolic disease increasing once more the spectrum of the non hemostatic function of coagulation/fibrinolytic parameters. Intensive glycemic control with insulin, decreasing weight, exercise, insulin sensitizer agents are effective in reducing the impact of this procoagulant state by favorably affecting platelet, coagulation and fibrinolysis dysfunction. Recognition of and therapy for a procoagulant state in obesity with insulin resistance is likely to result in a decrease in the atherothrombotic events that characterize these patients.

\section{References}

1. Ravussin E, Smith SR. Increased fat intake, impaired fat oxidation, and failure of fat cell proliferation result in ectopic fat storage, insulin resistance, and type 2 diabetes mellitus. Ann N Y Acad Sci 2002; 967: 363-78.

2. Ford ES, Giles WH, Dietz WH. Prevalence of the metabolic syndrome among US adults. Findings from the third national health and nutrition examination survey. JAMA 2002; 287: 356-9.

3. Isomaa B, Almgren P, Tuomi T, Forsen B, Lahti K, Nissen M, Taskinen MR, Groop L. Cardiovascular morbidity and mortality associated with the metabolic syndrome. Diabetes Care 2001; 24: 683-9.

4. Despres J P, Lamarche B, Mauriege P, Cantin B, Dagenais GR, Moorjani S, Lupien PJ. Hyperinsulinemia as an independent risk factor for ischemic heart disease. N Engl J Med 1996; 334: 952-7.

5. Juhan-Vague I, Alessi MC, Vague P. Increased plasma plasminogen activator inhibitor-1 levels. A possible link between insulin resistance and atherothrombosis. Diabetologia 1991; 34: 457-62.

6. Yudkin JS, Stehouwer CDA, Emeis JJ, Coppack SW. C-Reactive protein in healthy subjects: association with obesity, insulin resistance, and endothelial dysfunction. Arterioscler Thromb Vasc Biol 1999; 19: 9728.

7. Hotamisligil GS, Peraldi P. Budavari A, Ellis $\mathrm{R}$, White MF, Spiegelman BM. IRS-1-mediated inhibition of insulin receptor tyrosine kinase activity in TNF-alpha- and obesityinduced insulin resistance. Science 1996; 271 : 665-8.

8. Ziccardi P, Nappo F, Giugliano G, Esposito K, Marfella R, Cioffi M, D’Andrea F, Molinari AM, Giugliano D. Reduction of inflammatory cytokine concentrations and improvement of endothelial functions in obese women after weight loss over one year. Circulation 2002; 105: 9075-6.

9. Meigs JB, Mittleman MA, Nathan D, Tofler GH, Singer DE, Murphy-Sheehy PM, Lipinska I, D'Agostino RB, Wilson PW. Hyperinsulinemia, hyperglycemia and impaired hemostasis. The Framingham Offspring Study. JAMA 2000; 283: 221-8.

10. Juhan-Vague I, Thompson SG., Jespersen J. Involvement of the hemostatic system in the insulin resistance syndrome. A study of 1500 patients with Angina Pectoris. Arterioscler Thromb 1993; 13: 1865-73.

11. Westerbacka J, Yki-Jarvinen H, Turpeinen A,
Rissanen A, Vehkavaara S, Syrjala M, Lassila $\mathrm{R}$. Inhibition of platelet-collagen interaction: an in vivo action of insulin abolished by insulin resistance in obesity. Arterioscler Thromb Vasc Biol 2002; 22: 167-72.

12. Konstantinides S, Schafer K, Koschnick S, Loskutoff DJ. Leptin-dependent platelet aggregation and arterial thrombosis suggests a mechanism for atherothrombotic disease in obesity. J Clin Invest 2001; 108: 1533-40.

13. Juhan-Vague I, Morange PE, Renucci JF, Alessi MC. Fibrinogen, obesity and insulin resistance. Blood Coagulation Fibrinolysis 1999; 10 suppl 1: 25-28.

14. Mohamed-Ali V, Goodrick S, Rawesh A, Miles JM, Katz D, Yudkin JS, Coppack SW. Human subcutaneous adipose tissue secretes interleukin- 6 but not tumour necrosis factoralpha_in vivo. J Clin Endocrinol Metab 1997; 82: 4196-4200.

15. Folsom AR, Qamhieh HT, Wing RR, Jeffery RW, Stinson VL, Kuller LH, Wu KK. Impact of weight loss on plasminogen activator inhibitor (PAI-1), factor VII, and other hemostatic factors in moderately overweight adults. Arterioscler Thromb 1993; 13: 162-9.

16. Juhan-Vague I. Alessi MC, Morange PE. PAI1 , obesity and insulin resistance. In : G. 
Reaven and A. Laws (Ed) Contemporary Endocrinology : Insulin resistance. Humana Press Inc 1999; 317-332.

17. Kruszynska YT, Yu JG, Olefsky JM, Sobel BE. Effects of troglitazone on blood concentrations of plasminogen activator inhibitor 1 in patients with type 2 diabetes and in lean and obese normal subjects. Diabetes 2000; 49: 633-9.

18. Samad F, Uysal T, Wiesbrock S,Pandey M, Hotamisligil S, Loskutoff J. Tumor necrosis factor a is a key component in the obesitylinked elevation of plasminogen activator inhibitor 1. Proc Natl Acad Sci USA 1999; 96: 6902-7.

19. Alessi MC, Peiretti F, Morange P, Henry M, Nalbone G, Juhan-Vague I. Production of plasminogen activator inhibitor-1 by human adipose tissue: possible link between visceral fat accumulation and vascular disease. Diabetes 1997; 46: 860-7.

20. Morange PE, Lijnen HR, Alessi MC, Kopp F, Collen D, Juhan-Vague I. Influence of PAI-1 on adipose tissue growth and metabolic parameters in a murine model of diet-induced obesity.Arterioscler Thromb Vasc Biol 2000; 20: 1150-54.

21. Lijnen HR, Maquoi E., Morange P., Voros G., Van Hoef B., Kopp F., Collen D., Julian-Vague I., Alessi MC. Nutritionally induced obesity is attenuated in transgenic mice overexpressing plasminogen activator inhibitor-1. Arterioscler Thromb Vasc Biol. 2003, 23:78-84.

22. Juhan-Vague I, Pyke SDM, Alessi MC, Jespersen J, Haverkate F, Thompson SG. Fibrinolytic factors and the risk of myocardial infarction or sudden death in patients with angina pectoris. Circulation 1996; 94: $2057-$ 63.

23. Sobel BE, Woodcock-Mitchell J, Schneider DJ, Holt RE, Marutsuka K, Gold H. Increased plasminogen activator inhibitor type 1 in coronary artery atherectomy specimens from type 2 diabetic compared with nondiabetic patients: a potential factor predisposing to thrombosis and its persistence. Circulation 1998; 97: 2213-21.

24. Festa A, D'Agostino R, Tracy RP, Haffner SM. Elevated levels of acute-phase proteins and plasminogen activator inhibitor-1 predict the development of type 2 diabetes. The Insulin Resistance Atherosclerosis Study. Diabetes 2002; 51: 1131-7. 